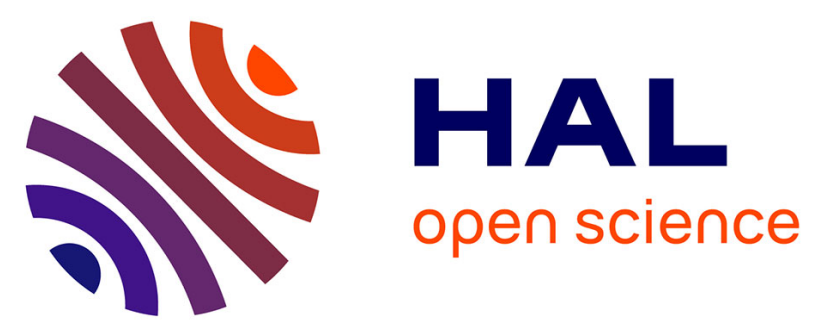

\title{
A three year follow-up study of gadolinium enhanced and non-enhanced regions in multiple sclerosis lesions using a multi-compartment T2 relaxometry model.
} Sudhanya Chatterjee, Olivier Commowick, Onur Afacan, Benoît Combès, Simon K. Warfield, Christian Barillot

\section{To cite this version:}

Sudhanya Chatterjee, Olivier Commowick, Onur Afacan, Benoît Combès, Simon K. Warfield, et al.. A three year follow-up study of gadolinium enhanced and non-enhanced regions in multiple sclerosis lesions using a multi-compartment T2 relaxometry model.. 2018. hal-01837974

\author{
HAL Id: hal-01837974 \\ https://hal.science/hal-01837974
}

Preprint submitted on 13 Jul 2018

HAL is a multi-disciplinary open access archive for the deposit and dissemination of scientific research documents, whether they are published or not. The documents may come from teaching and research institutions in France or abroad, or from public or private research centers.
L'archive ouverte pluridisciplinaire HAL, est destinée au dépôt et à la diffusion de documents scientifiques de niveau recherche, publiés ou non, émanant des établissements d'enseignement et de recherche français ou étrangers, des laboratoires publics ou privés. 


\title{
A three year follow-up study of gadolinium enhanced and non-enhanced regions in multiple sclerosis lesions using a multi-compartment $T_{2}$ relaxometry model
}

\author{
Sudhanya Chatterjee ${ }^{1 *}$, Olivier Commowick ${ }^{1}$, Onur Afacan ${ }^{2}$, Benoit Combes ${ }^{1}$, Simon K. \\ Warfield $^{2}$, Christian Barillot ${ }^{1}$, \\ 1 Univ Rennes, CNRS, Inria, Inserm, IRISA UMR 6074, VISAGES (Vision, action et \\ gestion des informations en santé) - ERL U 1228, F-35000 Rennes, France \\ 2 Computational Radiology Laboratory, Boston Children's Hospital, Department of \\ Radiology, 300 Longwood Avenue, WB215, Boston, MA 02115, USA \\ * sudhanya.chatterjee@irisa.fr
}

\begin{abstract}
Demyelination, axonal damage and inflammation are critical indicators of the onset and progress of neurodegenerative diseases such as multiple sclerosis (MS) in patients. Due to physical limitations of imaging such as acquisition time and imaging resolution, a voxel in a MR image is heterogeneous in terms of tissue microstructure such as myelin, axons, intra and extra cellular fluids and free water. We present a multi-compartment tissue model which estimates the water fraction (WF) of tissues with short, medium and high $T_{2}$ relaxation times in a $T_{2}$ relaxometry MRI voxel. The proposed method is validated on test-retest data of healthy controls. This model was then used to study longitudinal trends of the tissue microstructures for two sub-regions of the lesions: gadolinium enhanced $(E+)$ and non-enhanced $(L-)$ regions of MS lesions in $10 \mathrm{MS}$ patients over a period of three years. The water fraction values in $E+$ and $L-$ regions were found to be significantly different $(p<0.05)$ over the period of first three months. The results of this study also showed that the estimates of the proposed $T_{2}$ relaxometry model on brain tissue microstructures have potential to distinguish between regions undergoing active blood brain barrier breakdown from the other regions of the lesion.
\end{abstract}

\section{Introduction}

Magnetic resonance imaging (MRI) is one of the most widely used in-vivo imaging method for obtaining information on brain health. However, MRI voxels have limited resolution due to physical constraints. Use of advanced MRI techniques such as $T_{2}$ relaxometry and diffusion weighted MRI can address this issue by providing quantitative estimates on brain tissue microstructures such as myelinated and unmyelinated axons, brain fiber orientation etc 11. In patients with neurodegenerative diseases (such as multiple sclerosis (MS)), obtaining information on health of axons and myelin using in-vivo imaging techniques can improve our understanding of the current status and the progress of the disease in the patients. Obtaining this information can provide critical knowledge on the brain health.

The myelin, axons and fluids in the brain can be distinguished based on the nature of their water content. Myelin is a tightly wrapped structure around the axons. The water in myelin is very tightly bound to its surface. Hence myelin has a very short $T_{2}$ 
relaxation time compared to the other brain tissue structures. The axons, gray matter cells and other cells in the brain have a higher $T_{2}$ relaxation time than that of myelin but less than that of the fluids. Free fluids have the largest $T_{2}$ relaxation time. Hence, based on the $T_{2}$ relaxation time brain tissues can be broadly classified into three categories, short - , medium - and high $-T_{2}$ components 2, 3]. Since a voxel in a MR image of the brain is heterogeneous, there is a certain amount of the three $T_{2}$ components present in each voxel. In addition to these, in presence of an infection there might also be some fluid accumulation. Hence a quantitative metric that conveys information on the condition of these tissues can provide useful insights into the current brain tissue health.

Quantitative $T_{2}$ relaxometry MRI sequences offer the capability to distinguish tissues based on their $T_{2}$ relaxation times. $T_{2}$ relaxometry MRI has been used effectively to calculate the myelin water fraction using a variety of approaches. Whittall et al. and MacKay et al. 2, 4, obtained myelin water fraction using a multi-component $T_{2}$ model [5]. The multicomponent $T_{2}$ model describes the observed $T_{2}$ decay curve as a weighted sum of an arbitrary number of decay curves. The weight of each $T_{2}$ decay curve is obtained using non-negative least squares (NNLS). Whittall et al. obtained myelin water fraction of the brain tissues by assuming the $T_{2}$ values of water in myelin to be in the range of $10-55$ milliseconds 2 . The multi-component $T_{2}$ models select an arbitrary number of $T_{2}$ decay curves to model the observed decay signal [5]. For example, Whittall et al. 2] considered 80 logarithmically spaced values between 15 milliseconds and 2 seconds. Since the number of parameters to be estimated considerably outweighs the number of observations (i.e. number of echoes), some regularization is mandatory. However, the choice of the regularization term and its extent affects the fitting measure [6].

An alternate approach is a multi-compartment $T_{2}$ relaxometry model where the different $T_{2}$ water pools in brain tissues are modeled as a weighted mixture of pre-defined continuous functions. Stanisz and Henkelman 7 fitted two $T_{2}$ components (short and long $T_{2}$ in bovine optic nerve), each having a gaussian distribution on a logarithmic scale. Akhondi-Asl et al. proposed a framework to calculate myelin water fraction by modeling the $R_{2}$ space as a weighted mixture of inverse Gaussian distribution of fast-, medium - and slow- decaying components with respect to $T_{2}$ relaxation times [8]. Layton et al. proposed a maximum likelihood estimation approach to estimate the myelin water fraction values 9 . They obtained the Cramér-Rao lower bounds of the variables to establish the difficult nature of simultaneously estimating the model parameters and weights for each compartment for such models. In contrast to the multiple exponential $T_{2}$ fitting approach, these models do not suffer from the ill-posed weights estimation problem. In these models, the regularization is performed a priori rather than a posteriori.

In this work, we propose a method for computing water fractions corresponding to fast - , medium - and slow - decaying components with respect to $T_{2}$ relaxation times from $T_{2}$ relaxometry MRI data acquired using 2D multislice Carr-Purcell-Meiboom-Gill (CPMG) sequence. In this estimation framework, the $T_{2}$ space is modeled as a weighted mixture of three continuous probability density functions (PDF) representing the three $T_{2}$ compartments. For such models, the robustness and accuracy of the implementations to simultaneously estimate the weights and parameters of the distributions was found to be non-trivial and not reliable 9. We therefore chose to take advantage of the earlier studies performed in this field to fix the parameters of the distributions representing the $T_{2}$ relaxation components $3,10,11$. Hence only the estimation of weights remain, making the estimation model robust. In our work, we need to correct for the effect of the stimulated echoes due to imperfect refocusing (due to the $B_{1}$ inhomogeneities) that leads to errors in $T_{2}$ estimation [12]. The EPG algorithm is used to account for these 
stimulated echoes 13]. The field inhomogeneity $\left(B_{1}\right)$ is estimated numerically. Since the $T_{2}$ space is modeled as a weighted mixture of three continuous PDFs representing the three components, the proposed model does not include any regularization on the water fractions. The estimated weights of each compartment provide a quantitative estimate of the tissue microstructure in a voxel. Experiments were carried out to evaluate repeatability of the quantitative markers on healthy controls.

In the last part of this work, we show an application of the method on MS patients. We report observations on the evolution of the quantitative markers obtained from the model in enhancing (MS lesion regions undergoing active blood brain barrier breakdown) and non-enhancing MS lesion regions. Vavasour et al. studied the MWF and total water content in three new MS lesions in two patients over a year [14. Levesque et al. studied evolution of MWF and geometric mean of $T_{2}$ values of gadolinium enhancing lesions in five MS patients over a period of one year 15. Although MWF was found to be quite informative in suggesting changes in active lesions, the geometric mean $T_{2}$ values was sensitive to changes in the active lesions. Vargas et al. studied MWF of gadolinium enhancing and non-enhancing lesions [16]. Measurements were compared for two acquisitions with median duration of around 6 months between the scans. Authors found that contrary to the non-enhancing lesions, there was significant improvement in MWF of enhancing lesions between the two scans. Vargas et al. also mention that MWF is a combined measure of edema and demyelination [16]. The MWF is a relative measure and a change in its values should be studied in conjunction with the remaining water fraction measures. In our analysis, we observed and compared the change in water fractions of tissues with short, medium and high $T_{2}$ values in enhancing and non-enhancing regions of lesions in $10 \mathrm{MS}$ patients over a period of 3 years.

\section{Materials and methods}

\subsection{Estimation framework}

Signal model We model the $T_{2}$ space as a weighted mixture of three continuous probability density functions (PDF) representing the three $T_{2}$ relaxometry compartments. The compartments represent tissues with short, medium and high $T_{2}$ relaxation times. The weight of the $j$-th distribution is denoted by $w_{j}$. The weights are normalized such that $\sum_{j} w_{j}=1$. The signal of a voxel at the $i-$ th echo time $\left(t_{i}\right)$ is therefore given as:

$$
s\left(t_{i}\right)=M_{0} \sum_{j=1}^{3} w_{j}\left[\int_{0}^{\infty} f_{j}\left(T_{2} ; \mathbf{p}\right) \operatorname{EPG}\left(T_{2}, \triangle T E, i, B_{1}\right) d T_{2}\right]
$$

where $t_{i}=i \times \triangle T E$ and $\triangle T E$ is the echo spacing. Each $f_{j}\left(T_{2} ; \mathbf{p}\right)$ is the chosen PDF with parameters $\mathbf{p}$. As mentioned in the introduction, for robustness reasons, the PDF parameters are pre-selected and fixed keeping in mind histology findings reported in the literature [3 11. In Eq. (1), $\mathrm{M}_{0}$ is the magnetization constant. $E P G(\cdot)$ represents the stimulated echo computed at the time point $\left(t_{i}=i \times \triangle T E\right)$ using the EPG algorithm [13. $B_{1}$ is the field inhomogeneity.

Optimization Without any loss of generality, $M_{0}$ and $\left\{w_{j}\right\}_{j=1}^{3}$ in Eq. 11 can be combined into a single term, $\left\{\alpha_{j}\right\}_{j=1}^{3} \in \mathbb{R}^{+}$. The weight corresponding to each compartment can then be obtained as, $w_{j}=\alpha_{j} / \sum_{i} \alpha_{i}$ and $\mathrm{M}_{0}$ as $\sum_{i} \alpha_{i}$. The signal of 
the voxel at time $t_{i}$ is expressed as:

$$
s\left(t_{i}\right)=\sum_{j=1}^{3} \alpha_{j}\left[\int_{0}^{\infty} f_{j}\left(T_{2} ; \mathbf{p}\right) E P G\left(T_{2}, \triangle T E, i, B_{1}\right) d T_{2}\right]
$$

The parameters to be estimated in Eq. (2) are: $\left\{\left\{\alpha_{j}\right\}_{j=1}^{3}, B_{1}\right\}$. The optimization is thus formulated as a least square problem:

$$
\begin{aligned}
\left(\hat{\alpha}, \hat{B}_{1}\right) & =\underset{\alpha, B_{1}}{\arg \min } \sum_{i=1}^{m}\left(y_{i}-s\left(t_{i}\right)\right)^{2} \\
& =\underset{\alpha, B_{1}}{\arg \min }\left\|\mathbf{Y}-\boldsymbol{\Lambda}\left(B_{1}\right) \alpha\right\|_{2}^{2}
\end{aligned}
$$

where $m$ is the number of echoes; $\mathbf{Y} \in \mathbb{R}^{m}$ is the observed signal; $\alpha=\left\{\alpha_{1}, \alpha_{2}, \alpha_{3}\right\} \in \mathbb{R}^{+3}$. Each element of $\boldsymbol{\Lambda}$ $\left(\Lambda_{i j}=\lambda_{j}\left(t_{i} ; B_{1}\right) ; i=\{1, \ldots, m\}, j=\{1,2,3\}\right)$ in Eq. (3) is computed as:

$$
\lambda_{j}\left(t_{i} ; B_{1}\right)=\int_{0}^{\infty} f_{j}\left(T_{2} ; \mathbf{p}\right) E P G\left(T_{2}, \triangle T E, i, B_{1}\right) d T_{2}
$$

The parameters to be estimated in the least squares optimization problem stated in Eq. (3), $\alpha$ and $B_{1}$, are linear and non-linear in nature respectively. However they are linearly separable. Hence optimization for $\alpha$ and $B_{1}$ is performed alternatively until convergence is obtained in a desired error limit. In the first step, $\alpha$ is computed by non-negative least squares (NNLS) optimization 17 with a fixed $B_{1}$ value. In the next step, the weights computed in the first step are used to compute $B_{1}$ by a gradient free optimizer (BOBYQA). We choose to perform a numerical optimization to obtain $B_{1}$ as it does not have any closed form solution 13 . The integral in Eq. (4) also does not have a closed form solution. Hence the integral is computed using the Riemann sum approach by dividing the $T_{2}$ region into rectangles of finite width $(=0.33 \mathrm{~ms}$ in our case) over the range of $[0,2500] \mathrm{ms}$.

In this work, the PDFs to represent each $T_{2}$ relaxometry compartment $\left(\left\{f_{j}(\cdot)\right\}_{j=1}^{3}\right.$ in Eq. (1)) are chosen as Gaussian PDFs. The mean and standard deviations are chosen for the three compartments based on histology findings reported in the literature 3, 10, 11 and are set as $\mu=\{20,100,2000\}$ and $\sigma=\{5,10,80\}$ (all values in milliseconds). The proposed method was implemented in $\mathrm{C}++$ and is made available as an open source library Anima ${ }^{1}$

\subsection{Experimental Methods}

Repeatability is an important aspect of quantitative MRI techniques. In the first experiment we carried out test-retest experiments to assess the repeatability of the proposed method. An important motivation of this study is to gain more insights into the neurodegenerative disease phenomenon using the tissue microstructure information. Hence, in the second experiment we performed a 3-year follow-up study on 10 multiple sclerosis patients.

\subsubsection{Experiment-1: Repeatability test}

The objective of this experiment is to observe whether the proposed model is repeatable in terms of estimation of the microstructure maps. For that purpose, test retest $T_{2}$ relaxometry scans of 4 healthy controls were obtained to evaluate the repeatability of the proposed method.

\footnotetext{
${ }^{1}$ https://github.com/Inria-Visages/Anima-Public
} 
Healthy controls The age of the healthy controls was in the range of 26-32 years. 15 regions of interest (ROI) were marked in the brain for each healthy control over which the test and retest values of the compartments' water fractions were compared. An illustration of these ROI on a subject is shown in Fig. 1 .

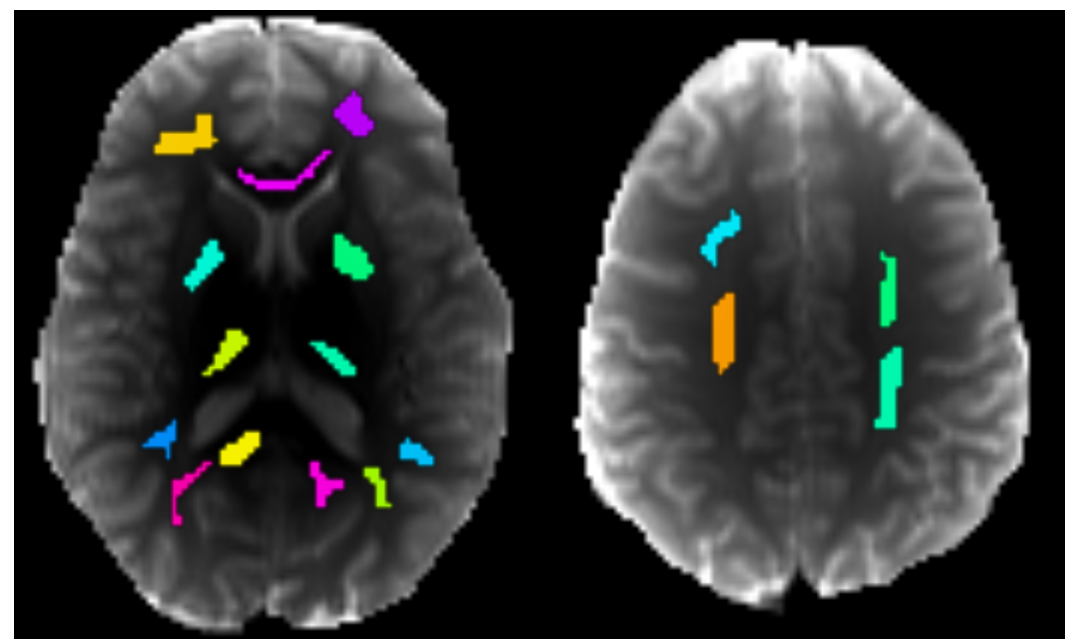

Fig 1. Assessing repeatability from test-retest data Test retest scans were performed for 4 healthy controls to study the repeatability of the proposed method. This figure shows the 15 regions which were marked on the healthy controls over which the repeatability was studied.

Acquisition details The details of the acquired data are as follows: 3T MRI scanner; 2D multislice CPMG sequence; 32 echoes; first echo at 9ms; $\triangle T E=9 \mathrm{~ms} ; T R$ $=3000 \mathrm{~ms}$; single slice acquisition; in plane resolution $=1.1 \mathrm{~mm} \times 1.1 \mathrm{~mm}$; slice thickness of $4 \mathrm{~mm}$; matrix size of $192 \times 192$; number of averages $=1$.

Analysis A Bland-Altman plot was used to observe the repeatability of the estimations over the ROIs. From the plot we obtained the mean deviation $\left(m_{d}\right)$ of the test retest values for the ROIs and checked whether there are noticeable systematic changes in the estimations. We further look at the limits of agreement (LoA) between the test retest estimations from the plot.

\subsubsection{Experiment-2: Application to Multiple Sclerosis (MS)}

Multiple sclerosis lesions are focal lesions and grow in a concentric manner [18]. In the early stages, brain tissues in the MS lesions undergo active blood brain barrier breakdown 18,19]. Surrounding the core of the lesion is the edema as a result of tissue inflammation due to ongoing tissue damage. As compared to the normal appearing brain matter, the entire MS lesion regions appear hyper-intense on $T_{2}$ weighted MRI. However, only the regions of the lesion undergoing active blood brain barrier breakdown appear hyperintense on $T_{1}$ weighted MRI acquired post Gd injection. Hence, lesions in active state have two regions, a region which actively undergoes blood brain barrier breakdown and the regions which do not.

The objective of this experiment is to study the evolution of compartments' water fractions in regions of lesions in MS patients undergoing active blood brain barrier breakdown and the regions which are not. In addition to that, we shall observe whether the water fraction values of the compartments for the two regions are in confirmation with the pathological knowledge of MS lesions. 
Patient Data We studied 10 MS patients over a period of 36 months. All patients included in this study had an episode of clinically isolated syndrome (CIS). The patient cohort comprised of an equal number of male and female subjects and their median age was 28 years. All participants of this study gave their written consent. Data was acquired at eight time points over a period of 36 months. An acquisition at the baseline was followed by acquisitions at 3, 6, 9, 12, 18, 24 and 36 months from the baseline. The lesions in patient scans were marked by an expert radiologist on $T_{2}$ weighted images at the baseline. All participants were informed and provided their written consent for the study.

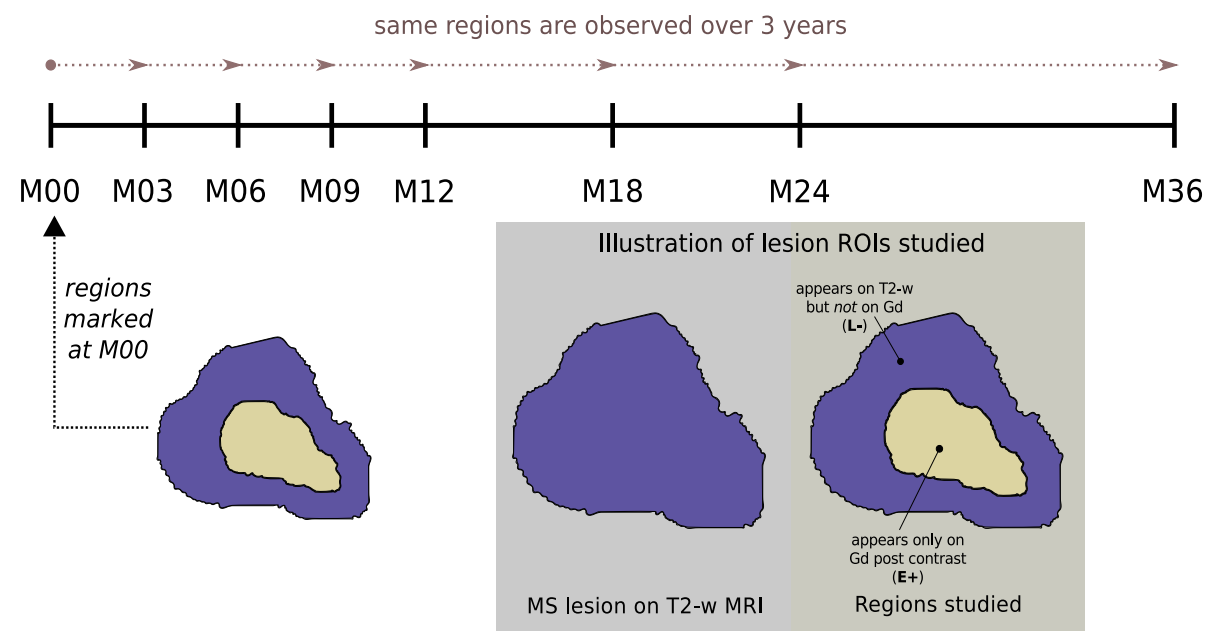

Fig 2. MS patient study design The MS lesions were marked on $T_{2}$ weighted on gadolinium post contrast $T_{1}$ spin echo MR images of the patients acquired at the first visit (i.e. M00). Eight scans are obtained from time of the first visit over a period of 36 months for each patient at intervals shown in the figure. The region of interest (ROI) marked at M00 is studied over the period of 36 months. Two ROIs are studied in this work: (i) region of the lesion which appears on the gadolinium post contrast $T_{1}$ weighted spin echo images. These are the regions of the lesions undergoing active blood brain barrier breakdown, (ii) lesion region appearing on $T_{2}$ weighted MR images but not on gadolinium post contrast images.

Acquisition details All data was acquired on a 3T MRI scanner. The acquisition details for the $T_{2}$ relaxometry data are as follows: $2 \mathrm{D}$ multislice CPMG sequence; 7 echoes; first echo at $13.8 \mathrm{~ms} ; \triangle T E=13.8 \mathrm{~ms} ; T R=4530 \mathrm{~ms}$; voxel dimensions $=1.3 \times 1.3 \times 3.0 \mathrm{~mm}^{3}$; spacing between slices of $3 \mathrm{~mm}$; matrix size of $192 \times 192$; number of averages $=1$; acquisition time $\approx 7$ minutes. A $T_{1}$ weighted scan was obtained post gadolinium injection to identify the lesions undergoing active blood brain barrier breakdown. The post Gadolinium injection $(0.1 \mathrm{mmol} / \mathrm{kg}$ gadopentate dimeglumine) acquisition details are as follows: transverse spin echo $T_{1}$ weighted images; voxel dimensions $=1.0 \times 1.0 \times 3.0 \mathrm{~mm}^{3}$; spacing between slices of $3 \mathrm{~mm}$; matrix size of $256 \times 256$; number of averages $=1$. The protocols were approved by the institutional review board of Rennes University Hospital.

Analysis In this study, we define two groups of MS lesions, (a) E+: lesion regions which appear hyper-intense on post-Gd injection $T_{1}$-weighted images and (b) $L$-: lesion regions appearing hyper-intense on $T_{2}$-weighted images only. Thus a lesion might have both $E+$ and $L-$ regions in it. An illustration of the lesion regions is shown in Fig. 2.

. 
The lesion ROIs are marked at baseline and the same region is observed over a period of three years. In the $10 \mathrm{MS}$ patients, we observed $229 \mathrm{~L}-$ and $25 \mathrm{E}+$ lesion regions. Since the lesions were marked on $T_{2}$ weighted images, all processed images were registered to the $T_{2}$ images using a block matching algorithm [20.21].

\section{Results}

\subsection{Experiment-1: Repeatability test}

The Bland-Altman (BA) plots for short, medium and high- $T_{2}$ water fraction estimates over the ROIs are shown in Fig. 3. BA plots are scatter plots between the average

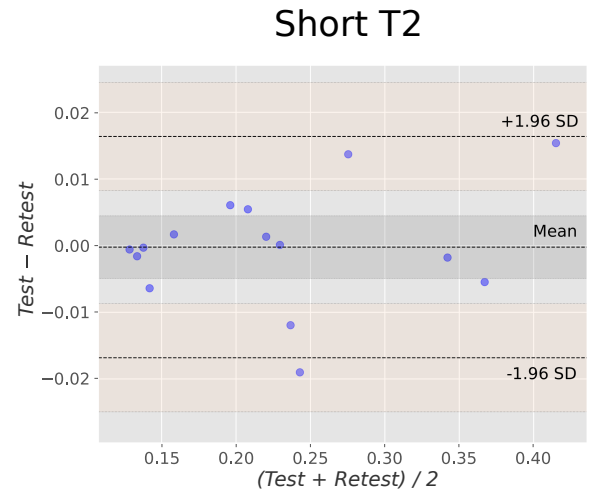

(a)

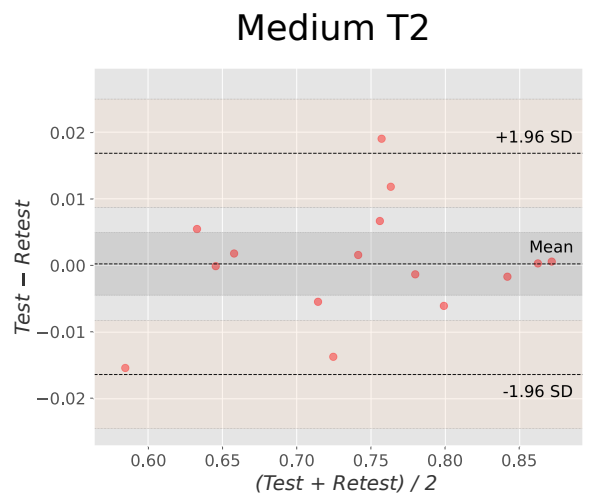

(b)

High T2

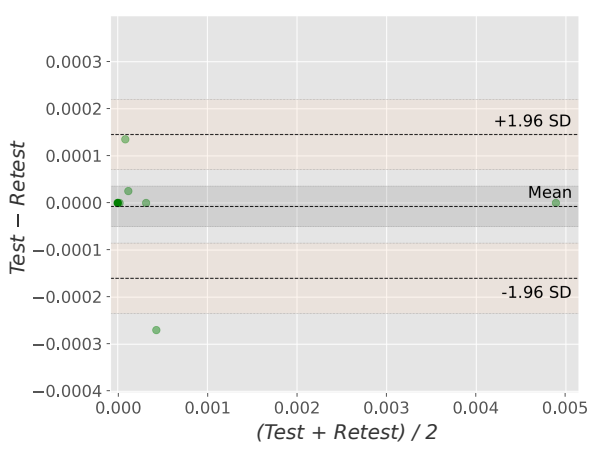

(c)

Fig 3. Bland-Altman plots comparing test-retest estimations Bland-Altman (BA) plots for (a) short, (b) medium and (c) high- $T_{2}$ water fraction estimations are shown here. 15 ROIs (see Fig. 1) are evaluated for repeatability of the estimated water fractions in 4 healthy controls. The mean level $\left(m_{d}\right)$ is the mean of the differences between test and retest values. The gray region around $m_{d}$ is its $95 \%$ confidence interval (CI). The limits of agreement (LoA) levels are computed as $m_{d} \pm 1.96 S D$, where $S D$ is the standard deviation of the difference in test and retest values. The yellow region around the LoAs are its 95\% CI. The BA plot statistics are summarized in Table 1

test-retest measurement and the difference between test-retest measurements. We measured the means of the estimated values of 15 ROIs in 4 healthy controls. The BA plots for short, medium and high- $T_{2}$ water fraction estimates for the 15 ROIs are shown in Fig. 3(a), 3(b) and 3(c) respectively. The plots shows the level of mean error $\left(m_{d}\right)$ 
observed. The gray area around the mean error level is its $95 \%$ confidence interval (CI). Along with $m_{d}$, the $m_{d} \pm 1.96 \times \sigma_{d}$ levels are also shown and are referred to as levels of agreement (LoA). $\sigma_{d}$ is the standard deviation associated with the errors observed in the test-retest measurements. LoA is thus an empirical estimate of the range around $m_{d}$ within which $95 \%$ of the differences are expected to exist. The $95 \%$ CI of the LoAs for each plot is shown with a yellow shade around LoA in the plots. The BA plot statistics are summarized in Table1. From Fig. 3, we observe that the mean bias of difference between the test-retest ROI mean values $\left(m_{d}\right)$ is close to zero for short, medium and high- $T_{2}$ water fraction estimates. For all three water fraction estimations, the zero level lies comfortably inside the $95 \% \mathrm{CI}$ of the $m_{d}$. The test retest differences lie within the LoA and its $95 \%$ CI.

Table 1. Bland-Altman plots statistics for repeatability experiment

\begin{tabular}{lccc}
\hline & Mean bias $\left(m_{d}\right)$ & $95 \%$ CI around $m_{d}$ & Limits of agreement $($ LoA $)$ \\
\hline Short-T2 water fraction & $-2.27 \times 10^{-4}$ & {$\left[-4.92 \times 10^{-3}, 4.46 \times 10^{-3}\right]$} & $1.65 \times 10^{-2}$ \\
Medium- $T_{2}$ water fraction & $2.34 \times 10^{-4}$ & {$\left[-4.45 \times 10^{-3}, 4.91 \times 10^{-3}\right]$} & $1.65 \times 10^{-2}$ \\
High- $T_{2}$ water fraction & $-0.07 \times 10^{-4}$ & {$\left[-0.11 \times 10^{-3}, 0.09 \times 10^{-3}\right]$} & $0.01 \times 10^{-2}$ \\
\hline
\end{tabular}

This table summarizes the statistics of the Bland-Altman (BA) plots shown in Fig. 3 for short, medium and high- $T_{2}$ water fraction estimates.

\subsection{Experiment-2: Application to Multiple Sclerosis (MS)}

In this experiment we observed and compared the evolution of water fraction maps of the three compartments between $E+$ and $L-$ MS lesion regions in 10 patients over a period of 3 years. We observed the water fraction values for $E+$ and $L-$ at each scan point. In addition to it, we also observed the change in the water fraction values (for $E+$ and $L-)$ between consecutive scans which is computed as: $\Delta w_{f, i}=$ $\left(w_{f_{\text {scan }}+1}-w_{f_{\text {scan }_{i}}}\right)$. Hence positive values indicate a gain in the water fraction values between consecutive scans. The $E+$ and $L$ - group difference analysis was performed using Mann-Whitney U test.

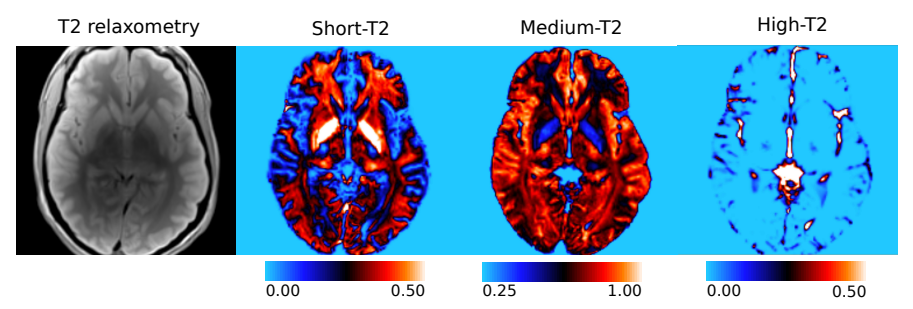

(a) Healthy subject

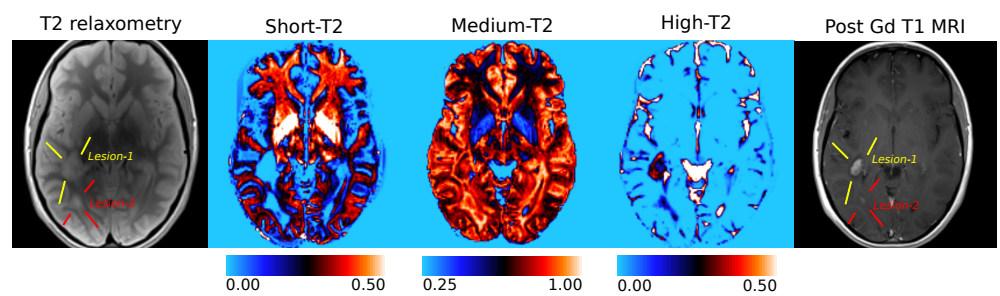

(b) MS patient

Fig 4. Healthy subject and MS patient A comparison between water fraction maps for (a) healthy subject and (b) MS patient is shown.

An example illustrating the comparison between the water fraction maps for a 
healthy control and MS patient is shown in Fig. 4. Lesion-1 in the MS patient has a large active core, whereas a very small region of lesion- 2 is active. Both lesions show indications of extensive demyelination. The medium- $T_{2}$ and high- $T_{2}$ water fraction maps show varying trends among the lesions, and also between the regions of the lesion undergoing active blood brain barrier breakdown and otherwise.

Short $T_{2}$ water fraction Results for short- $T_{2}$ water fraction $\left(w_{s}\right)$ maps is shown in Fig. 5. The $w_{s}$ values of the $E+$ and $L-$ lesions at all time points is shown in Fig. 5(a). The $L$ - lesion regions are significantly associated with higher $w_{s}$ values as compared to $E+$ at M00 $(p=0.014)$. However, the $w_{s}$ distribution of $E+$ and $L-$ regions at the end of 3 years are similar. The results of the change in $w_{s}$ values $\left(\Delta w_{s}\right)$ between consecutive scans in shown in Fig. 5(b). Largely negative $\Delta w_{s, 0}$ values for $E+$ lesion regions suggests increased $w_{s}$ values between M00 and M03. After M06 we observed less changes in $w_{s}$ values in $E+$ lesion regions. The observed change in $w_{s}$ values for the $L-$ lesions regions was very less throughout the 3 year period. Only $\Delta w_{s, 0}$ values $E+$ and $L$ - were significantly different $(p=0.009)$.

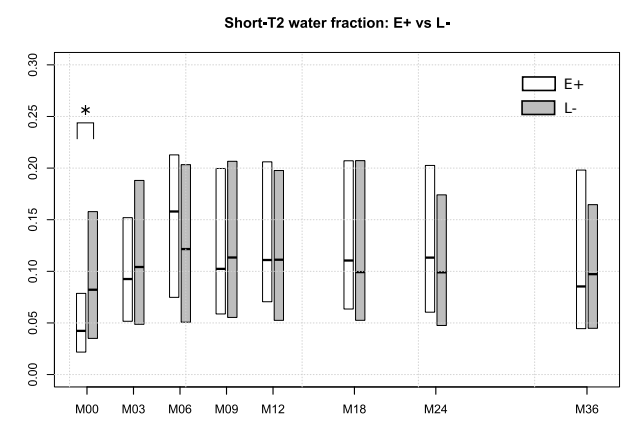

(a)

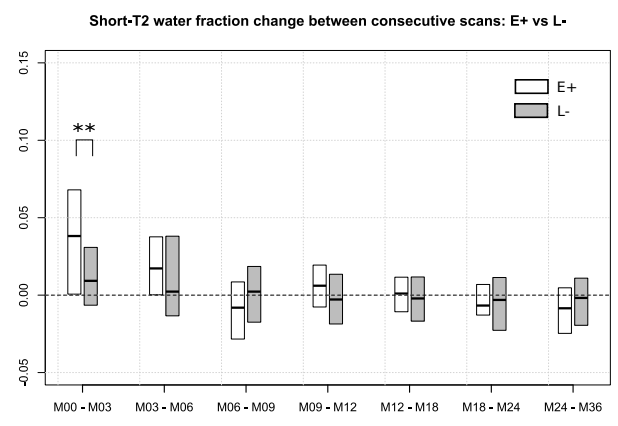

(b)

Fig 5. Evolution of short $T_{2}$ water fraction values over 3 years The plots in this figure show the median and upper and lower quartile levels of the data. The short- $T_{2}$ water fraction value $\left(w_{s}\right)$ at each scan is shown in Fig. 5 (a). The change in $w_{s}$ between consecutive scans is shown in Fig. 5(b). Significant group differences between groups are shown using ${ }^{*}(p<0.05)$ and ${ }^{* *}(p<0.01)$.

Medium- $T_{2}$ water fraction The evolution of the medium- $T_{2}$ water fraction $\left(w_{m}\right)$ in $E+$ and $L-$ lesion regions is shown in Fig. 6(a). Although the $w_{m}$ values for both groups reduce slightly at the end of 3 years, there is no evidence of difference between $E+$ and $L$ - with respect to the change in $w_{m}$ values between successive scans (refer Fig. 6(b)).

High- $T_{2}$ water fraction The high- $T_{2}$ water fraction $\left(w_{h}\right)$ values for $E+$ and $L-$ lesion regions are shown in Fig. 7(a). The $E+$ lesion regions are significantly associated with a higher value of $w_{h}$ as compared to the $L$ - population at M00 $(p=0.002)$.

Largely negative $\Delta w_{h, 0}$ values (refer Fig. 7(b)) for $E+$ lesion regions suggest a decrease in their $w_{h}$ values between M00 and M03. Subsequently, $E+$ lesion regions undergo negligible change between consecutive scans. $L$ - lesion regions show negligible change in their $w_{h}$ values throughout the period of the study. The change in $w_{h}$ values were found to be significantly different between $E+$ and $L$ - lesion regions for scan periods M00-M03, M03-M06 and M06-M09 with p-values of 0.008, 0.011 and 0.11 respectively.

It is also important to observe the effect size of the datasets when observing the p-values for group differences. Whereas the p-value conveys information on the strength 


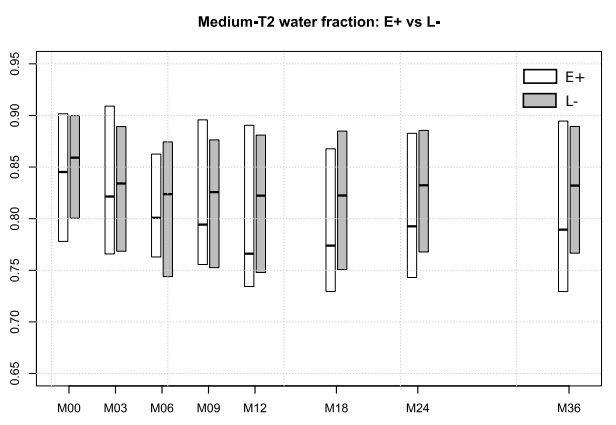

(a)

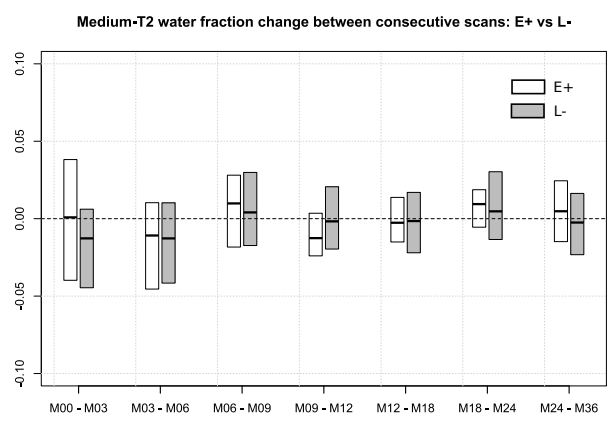

(b)

Fig 6. Evolution of medium $T_{2}$ water fraction values over 3 years The plots in this figure show the median and upper and lower quartile levels of the data. The medium- $T_{2}$ water fraction value $\left(w_{m}\right)$ at each scan is shown in Fig. 6(a). The change in $w_{m}$ between consecutive scans is shown in Fig. 6(b). Significant group differences between groups are shown using ${ }^{*}(p<0.05)$ and ${ }^{* *}(p<0.01)$.

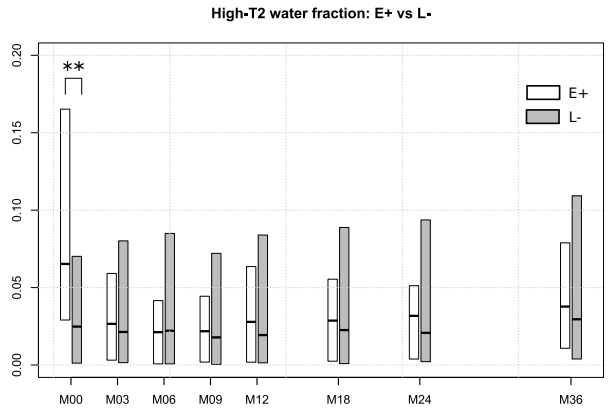

(a)

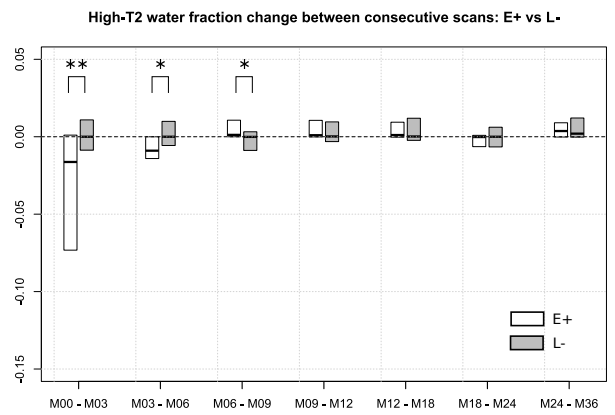

(b)

Fig 7. Evolution of high $T_{2}$ water fraction values over 3 years The plots in this figure show the median and upper and lower quartile levels of the data. The high- $T_{2}$ water fraction value $\left(w_{h}\right)$ at each scan is shown in Fig. 7)(a). The change in $w_{h}$ between consecutive scans is shown in Fig. 7(b). Significant group differences between groups are shown using ${ }^{*}(p<0.05)$ and ${ }^{* *}(p<0.01)$.

Table 2. Common language effect size and p-values

\begin{tabular}{lcc}
\hline \multicolumn{1}{c}{ Value } & $\boldsymbol{p}$-value & $\boldsymbol{C L}$ effect size \\
\hline Short- $T_{2}$ water fraction $\left(w_{s}\right)$ at M00 & 0.014 & $65.05 \%$ \\
$\Delta w_{s}$ between M00 and M03 & 0.009 & $34.25 \%$ \\
High- $T_{2}$ water fraction $\left(w_{h}\right)$ at M00 & 0.002 & $31.51 \%$ \\
$\Delta w_{h}$ between M00 and M03 & 0.008 & $66.08 \%$ \\
$\Delta w_{h}$ between M03 and M06 & 0.011 & $65.51 \%$ \\
$\Delta w_{h}$ between M06 and M09 & 0.011 & $34.55 \%$
\end{tabular}

The common language (CL) effect size are reported for the measurements which were significantly different for $E+$ and $L-$. In this work, the CL effect size denotes the percentage of times a randomly selected sample from $L-$ is greater than a random selection from $E+$ group.

of the water fraction values to reject the null hypothesis, the effect size is a measure of the magnitude of the difference. We used the common language (CL) effect size statistic and the associated p-values to observe the group differences $22[23]$. The CL effect size 257 258 259 
for the groups that were found to be significantly different from the Mann-Whitney U test is shown in Table 2. In this work, the CL effect size value denotes the percentage of times the $w_{f}$ value of $L$ - is higher than $E+$ when both samples are selected at random from each group. It can hence be interpreted as the probability of superiority of $L-$ over $E+$ for a measurement.

\section{Discussion}

The test-retest experiment results show that the quantitative MRI markers estimated by the proposed method is repeatable. For all the markers estimated, the zero level was comfortably inside the $95 \%$ confidence interval of the mean difference observed between the test and retest values of the marker for the 15 ROIs (refer the Bland-Altman plots in Fig. 22). Hence there are no noticeable systematic changes in the estimated markers for the test and retest data 24].

In Section 3.2 the evolution of water fraction markers in MS lesion regions which are undergoing active blood brain barrier breakdown are compared to the MS lesion regions in the later stages. At the baseline scan, the $E+$ lesion regions are prone to having lower short- $T_{2}$ water fraction values as compared to $L-$ regions $(p=0.014, \mathrm{CL}=$ $65.05 \%$ ). This might indicate that regions of the lesion undergoing active blood brain barrier breakdown have undergone greater demyelination. However, there seems to be no significant difference between the two groups with respect to the short- $T_{2}$ water fraction values for all scans three months after the baseline. The $E+$ lesion regions tend to have significantly higher values for high- $T_{2}$ water fraction as compared to $L-$ at the baseline scan $(p<0.01, \mathrm{CL}=31.51 \%)$. The demyelination of MS lesions is

accompanied by inflammation due to increased macrophage intervention 19]. This might explain the low and high values of short- $T_{2}$ and high- $T_{2}$ water fraction values observed at the baseline scan for $E+$ lesion regions. The gain in short- $T_{2}$ water fraction values for $E+$ lesion regions between the scans at M00 and M03 is significantly greater than that for $L-(p<0.01, \mathrm{CL}=34.25 \%)$. The increase in short- $T_{2}$ values in this period is accompanied by a considerable drop in high- $T_{2}$ values for the $E+$ lesion regions. The drop in high- $T_{2}$ water fraction values between the baseline and the next scan for $E+$ is significantly larger than that of $L-(p<0.01, \mathrm{CL}=66.08 \%)$. All $E+$ lesion regions incur a decrease in high- $T_{2}$ values over the first six months of observation. Hence, the observations from the change in water fraction values between consecutive scans are: (i) although both groups show indications of remyelination, $E+$ lesion regions undergo significantly greater remyelination as compared to $L$ - between the baseline and scan at M03, (ii) there is a considerable reduction of inflammation in $E+$ over the first three months but $L$ - show little or no change in this aspect. The myelination activity of $E+$ and $L-$ is similar three months after baseline. However, the inflammation activity seems to continue for 9 months from baseline scan time. Some $L$ - lesion regions observed at the baseline scan would have been in the $E+$ stage at some point of time. This explains the similar water fraction values for $E+$ and $L-$ by the end of three years. Unlike short- $T_{2}$ and high- $T_{2}$ water fraction values, the medium- $T_{2}$ water fraction values for $E+$ and $L$ - lesion regions never show any significant difference, which might be attributed to the fact that in terms of $T_{2}$ values considered, the medium- $T_{2}$ water pool is highly heterogeneous. It conveys information on unmyelinated axons, glia, interstitial and extra-cellular matters [10].

Our study on MS patients has certain limitations. First, the clinical data available was not of the highest quality possible due to acquisition time constraints in a clinical setting. $T_{2}$ relaxometry data with a higher number of echoes and shorter echo times are favorable for multi-compartment models. Second, the time gap between the first and second scan was three months. A shorter interval between successive acquisitions would 


\section{Conclusion}

In this work we proposed a multi-compartment $T_{2}$ relaxometry model to obtain quantitative estimates on brain tissues with short, medium and high $T_{2}$ relaxation times. Test retest experiment results showed that the proposed method does not suffer from any noticeable systematic changes in terms of the markers estimated. The study of the evolution of multi-compartment $T_{2}$ relaxometry markers on $10 \mathrm{MS}$ patients over a period of 3 years had two important observations: (i) the markers have the potential to distinguish between gadolinium enhanced and non-enhanced regions in MS lesions and (ii) both lesion regions have similar water fraction values by the end of the third year and show little distinction after 3 months from baseline scan. The later observation from the longitudinal study shows that the biomarkers obtained from this model explains the MS lesion evolution along the lines reported in the pathological and radiological studies on MS lesions [18 19]. The former observation on the other hand motivates us to investigate methods by which biomarkers from multi-compartment models based on advanced MRI techniques can help distinguish between lesion regions undergoing active blood brain barrier breakdown without injection of contrast enhancers in MS patients, which we shall investigate in our future studies.

\section{Acknowledgments}

The clinical MS study was supported by a grant from Fondation pour l'Aide à la Recherche sur la Sclérose en Plaques (ARSEP). The authors acknowledge Neurinfo MRI research facility for logistic support and MRI data acquisition. This research was supported in part by the grants NIH R01 EB019483 and NIH R01 NS079788.

\section{References}

\section{References}

1. Tofts P. Quantitative MRI of the brain: measuring changes caused by disease. John Wiley \& Sons; 2005. p. 477-500.

2. Whittall KP, MacKay AL, Graeb DA, Nugent RA, Li DK, Paty DW. In vivo measurement of $\mathrm{T} 2$ distributions and water contents in normal human brain. Magnetic resonance in medicine. 1997;37(1):34-43. doi:10.1002/mrm.1910370107.

3. MacKay AL, Laule C. Magnetic Resonance of Myelin Water: An in vivo Marker for Myelin. Brain Plasticity. 2016;2(1):71-91.

4. MacKay A, Whittall K, Adler J, Li D, Paty D, Graeb D. In vivo visualization of myelin water in brain by magnetic resonance. Magnetic Resonance in Medicine. 1994;31(6):673-677. doi:10.1002/mrm.1910310614.

5. Whittall KP, MacKay AL. Quantitative interpretation of NMR relaxation data. Journal of Magnetic Resonance (1969). 1989;84(1):134-152.

6. Does MD. Inferring brain tissue composition and microstructure via MR relaxometry. NeuroImage. 2018; 
7. Stanisz GJ, Henkelman RM. Diffusional anisotropy of T2 components in bovine optic nerve. Magnetic resonance in medicine. 1998;40(3):405-410.

8. Akhondi-Asl A, Afacan O, Balasubramanian M, Mulkern RV, Warfield SK. Fast myelin water fraction estimation using 2D multislice CPMG. Magnetic Resonance in Medicine. 2015;c. doi:10.1002/mrm.26034.

9. Layton KJ, Morelande M, Wright D, Farrell PM, Moran B, Johnston LA. Modelling and Estimation of Multicomponent $T_{2}$ Distributions. IEEE transactions on medical imaging. 2013;32(8):1423-1434.

10. Lancaster JL, Andrews T, Hardies LJ, Dodd S, Fox PT. Three-pool model of white matter. Journal of Magnetic Resonance Imaging. 2003;17(1):1-10.

11. Laule C, Vavasour IM, Kolind SH, Li DKB, Traboulsee TL, Moore GRW, et al. Magnetic resonance imaging of myelin. Neurotherapeutics. 2007;4(3):460-484. doi:10.1016/j.nurt.2007.05.004.

12. Crawley A, Henkelman R. Errors in T2 estimation using multislice multiple-echo imaging. Magnetic resonance in medicine. 1987;4(1):34-47.

13. Prasloski T, Mädler B, Xiang QS, MacKay A, Jones C. Applications of stimulated echo correction to multicomponent T2 analysis. Magnetic Resonance in Medicine. 2012;67(6):1803-1814. doi:10.1002/mrm.23157.

14. Vavasour I, Laule C, Li D, Oger J, Moore G, Traboulsee A, et al. Longitudinal changes in myelin water fraction in two MS patients with active disease. Journal of the neurological sciences. 2009;276(1):49-53.

15. Levesque IR, Giacomini PS, Narayanan S, Ribeiro LT, Sled JG, Arnold DL, et al. Quantitative magnetization transfer and myelin water imaging of the evolution of acute multiple sclerosis lesions. Magnetic resonance in medicine. 2010;63(3):633-640.

16. Vargas WS, Monohan E, Pandya S, Raj A, Vartanian T, Nguyen TD, et al. Measuring longitudinal myelin water fraction in new multiple sclerosis lesions. NeuroImage: Clinical. 2015;9:369-375.

17. Lawson CL, Hanson RJ. Solving least squares problems. SIAM; 1995.

18. Guttmann C, Ahn SS, Hsu L, Kikinis R, Jolesz FA. The evolution of multiple sclerosis lesions on serial MR. American journal of neuroradiology. 1995;16(7):1481-1491.

19. Lassmann H, Bruck W, Lucchinetti C. Heterogeneity of multiple sclerosis pathogenesis: Implications for diagnosis and therapy. Trends in Molecular Medicine. 2001;7(3):115-121. doi:10.1016/S1471-4914(00)01909-2.

20. Ourselin S, Roche A, Prima S, Ayache N. Block matching: A general framework to improve robustness of rigid registration of medical images. In: Medical Image Computing and Computer-Assisted Intervention-MICCAI 2000. Springer; 2000. p. CH373-CH373.

21. Commowick O, Wiest-Daesslé N, Prima S. Block-matching strategies for rigid registration of multimodal medical images. In: Biomedical Imaging (ISBI), 2012 9th IEEE International Symposium on. IEEE; 2012. p. 700-703. 
22. McGraw KO, Wong S. A common language effect size statistic. Psychological bulletin. 1992;111(2):361.

23. Grissom RJ, Kim JJ. Effect sizes for research: A broad practical approach. Lawrence Erlbaum Associates Publishers; 2005.

24. Lexell JE, Downham DY. How to assess the reliability of measurements in rehabilitation. American journal of physical medicine \& rehabilitation. 2005;84(9):719-723. 